\title{
Risk Prediction Model for Cardiac Implantable Electronic Device Implantation After Transcatheter Aortic Valve Replacement in Patients with Pre-Existing Atrial Fibrillation
}

\author{
Heather Wheat ${ }^{1}$, Takahiro Tshusima ${ }^{1}$, Sadeer Al-Kindi ${ }^{1}$, Fahd Nadeem ${ }^{1}$, Guilherme \\ Attizzani $^{1}$, Yakov Elgudin ${ }^{1}$, Alan Markowitz ${ }^{2}$, Marco Costa ${ }^{1}$, Daniel Simon ${ }^{1}$, Mauricio \\ Arruda $^{3}$, Judith Mackall ${ }^{3}$, and Sergio Thal ${ }^{1}$ \\ ${ }^{1}$ Case Western Reserve University Hospital \\ ${ }^{2}$ Case Western Reserve University \\ ${ }^{3}$ University Hospitals Harrington Heart and Vascular Institute, Case Western Reserve \\ University
}

July 7, 2020

\begin{abstract}
Introduction High-grade atrioventricular block (AVB) requiring cardiac implantable electronic device (CIED) following transcatheter aortic valve replacement (TAVR) is an important post-procedural complication. Risk prediction models have been developed to identify patients in sinus rhythm at risk for CIED placement following TAVR. Atrial fibrillation (AF) is frequently present in patients referred for TAVR and no risk prediction model has been developed for these patients. This study was undertaken to assess the accuracy of utilizing a previously validated risk prediction model in patients with pre-existing atrial fibrillation (1). Methods and Results This single center, retrospective study analyzed patients with pre-existing AF who underwent TAVR from July 2015 to November 2019. A summative risk prediction score was calculated using a previously described risk prediction model based on the use of a self-expanding valve (1 point), hypertension (1 point), and right bundle branch block on baseline electrocardiogram (2 points), and omitting pre-existing first degree AVB. The model demonstrated good predictive accuracy with an ideal linear regression in calibration plot $(\mathrm{R} 2=0.778)$ and AUC in ROC of $0.652(95 \%$ confidence interval $0.558-0.745)$. Conclusions Our previously validated risk prediction model accurately predicts the risk of post-TAVR CIED implantation in patients with pre-existing AF.
\end{abstract}

\section{Introduction}

Since its introduction in 2002, the use of transcatheter aortic valve replacement (TAVR) has dramatically increased. Originally indicated for high-risk surgical patients with symptomatic severe aortic stenosis (AS), TAVR has subsequently been shown to be non-inferior to conventional surgical aortic valve replacement (SAVR) in patients with low-intermediate surgical risk (2-5).

Despite advanced techniques and increased procedural expertise, the implantation of post-procedural cardiac implantable electronic device (CIED) remains an important post-procedural complication (6-10). Most TAVR patients requiring CIED develop acute high-grade atrioventricular block (AVB) in the immediate post-procedural period, and this contributes to a prolonged hospitalization and delayed post-procedural recovery. Other patients develop late-onset high-grade conduction defects after the initial hospitalization. Because patients with atrial fibrillation (AF) are often on long-term anticoagulation, they may experience a greater risk of trauma after mechanical fall or cardiac syncope. In addition, low risk surgical patients may have a high risk of heart block with TAVR, and SAVR may be a better option. Use of risk prediction models 
can help inform both patients and physicians about the risk of post procedural cardiac device. For these reasons, the pre-procedural risk stratification is essential for the conventional TAVR practice, especially in patients with $\mathrm{AF}$, and there are no previously published risk prediction models for these patients.

Several risk calculators have been developed to identify patients at risk for post-TAVR CIED implantation $(1,11-12)$. Kiani et al. reported the Emory Risk Score, however this model was only applicable to patients with balloon-expandable valve (Edwards SAPIEN 3), not for those receiving self-expanding prostheses. Also, the diagnostic accuracy of the Emory Risk Score has not been validated for patients with pre-existing AF. To our knowledge, no studies have reported the risk of post-TAVR CIED requirement in patients with preexisting AF. Our research team suggested a simple prediction model that was composed of pre-existing AVB (1 point), hypertension (1 point), self-expanding valve use (1 point), and pre-existing right bundle branch block (RBBB; 2 points), respectively (1). This model was internally validated for patients in normal sinus rhythm requiring both balloon-expandable and self-expanding prostheses (AUC on ROC $=0.693$ ). The goal of this study is to validate the diagnostic accuracy of our previously reported risk prediction model in patients with pre-existing AF.

\section{Methods}

\section{Study Design}

This is a single center, retrospective study of patients who underwent TAVR from July 2015 through November 2019 at University Hospitals Cleveland Medical Center. Patients with no AF prior to TAVR and/or pre-existing CIED, such as permanent pacemaker (PPM), implantable cardioverter-defibrillator (ICD), or cardiac resynchronization therapy (CRT) were excluded from the study. In addition, the first generation TAVR prosthesis, such as CoreValve (Medtronic, Minneapolis, Minnesota, USA), Edwards SAPIEN Valve (Edwards Lifesciences, Irvine, California, USA), and Edwards SAPIEN XT were also excluded from this study, as the newer generation valves are associated with less vascular complications and aortic regurgitation (13-14). Patient characteristics, comorbidities, pre-procedural electrocardiogram (ECG) and imaging, intraprocedural data, and the incidence of post-procedural CIED implantations were recorded. All patients had pre-existing AF recorded on at least one pre-procedural ECG. This study utilized a dataset extracted from the TAVR research registry, which was approved by the Institutional Review Board at University Hospitals Cleveland Medical Center. Informed consent was obtained from all patients.

\section{Summary of our previously reported model}

The development of the risk-prediction model for CIED after TAVR has been previously described (1). In brief, 888 patients were randomly divided into two groups, a derivation cohort and validation cohort (507 and 381 cases, respectively). Multivariate logistic regression was used to determine risk factors associated with post-TAVR CIED for the development of the prediction model, which was subsequently verified by the internal validation cohort. Components of the risk prediction model include hypertension (1 point), first-degree AVB on pre-procedural ECG (1 point), use of a self-expanding valve (1 point), and right bundle branch block (RBBB) identified on pre-procedural ECG (2 points). In this retrospective subgroup analysis of patients with AF, the element of pre-existing AVB was automatically assigned to zero points due to the inability to determine AVB in the setting of the AF on ECG. The remaining three variables were utilized in the newly included AF patients before TAVR and were fitted to the risk prediction model without any changes.

\section{TAVR Procedure}

Patient selection for TAVR was decided by our heart team including interventional cardiologists and 2 cardiac surgeons per Centers for Medicare and Medicaid Services policy (15). The TAVR prothesis type, sheath size, vascular access, and pre-balloon dilation were decided by our TAVR heart team. The indication for post-procedure CIED implantation was determined after consultation with a cardiac electrophysiologist. A protocol was developed in 2017, similar to that presented in Circulation in 2017, that assisted in the decision for CIED placement (7). 


\section{Statistical Analysis}

The risk score was calculated for all included patients with pre-existing AF. Categorical variables were compared with Pearson's Chi-square test or Fisher's exact test. Mann-Whitney test was used for quantitative variables with a non-normal distribution. An area under the curve (AUC) in receiver-operating characteristic (ROC) and calibration plot were used to assess the diagnostic accuracy of the risk prediction model for the sample. All p-values were 2 -sided and p-values $<5 \%$ were considered significant. Statistical analysis was performed with IBM SPSS Statistics software (version 25; IBM Corp., Armonk, New York).

\section{Results}

\section{Patient characteristics}

Between July 2015 and November 2019, 240 patients with pre-existing AF underwent TAVR with new generation prostheses. Of those, 47 required post-TAVR CIED placement (19.5\%). Baseline characteristics of the patients who did or did not require post-procedural CIED are reported in Table 1. Chronic kidney disease and prior coronary artery bypass graft (CABG) surgery were more common in the CIED group ( $57.4 \%$ vs $35.8 \% ; \mathrm{p}=0.003$ and $29.8 \%$ vs $14.5 \% ; \mathrm{p}=0.010$, respectively). On baseline ECG, RBBB was associated with higher post-procedural CIED placement, and the baseline QRS duration (milliseconds) was also statistically different between the two groups ( $42.6 \%$ vs $11.9 \% ; \mathrm{p}<0.001$ and $116.61 \pm 25.75$ vs $104.84 \pm$ 26.13; $\mathrm{p}=0.003$, respectively). Similarly, new left bundle branch block (LBBB) post-procedurally was higher in the CIED group, and width of the post-TAVR QRS was wider in patients requiring new CIED after TAVR (29.8\% vs $15.5 \% ; \mathrm{p}<0.001$ and $135.93 \pm 31.07$ vs $118.75 \pm 27.01 ; \mathrm{p}=0.004)$. Baseline echocardiographic findings, including the peak velocity (Vmax; meters/second) across the aortic valve and mean aortic valve pressure gradient (AVPG, in $\mathrm{mmHg}$ ) where significantly different between groups $(3.61 \pm 0.74$ vs $3.92 \pm$ $0.81 ; \mathrm{p}=0.016$ and $32.27 \pm 13.37$ vs $38.42 \pm 15.01 ; \mathrm{p}=0.005$, respectively). Use of a self-expandable valve were not statistically different between the two groups.

\section{Performance of our suggested model in patients with pre-existing AF}

The performance of the previously developed prediction model was tested in this study sample. Patients were divided into subgroups based on the number of points (0-4) calculated using the prediction model. Patient distribution of each scored group was similar to those with pre-existing AF when compared with our previous validation cohort (Figure 1). The highest incidence of post-TAVR CIED implantation was $47.4 \%$ in patients with a sum of 4 points, as opposed to the highest incidence in our previously reported validation cohort $(57.5 \%$ or $100 \%$ in patients with a sum of 4 or 5 points, respectively; Table 2$)$. The model demonstrated good predictive accuracy of an ideal linear regression in calibration plot $\left(\mathrm{R}^{2}=0.778\right)$ and AUC in ROC of 0.652 (95\% confidence interval 0.558 - 0.745; Figure 2).

\section{Discussion}

Identification of patients that will require CIED placement after TAVR remains vitally important as postTAVR conduction disturbances continue to remain an important complication (7). Not only are patients subject to an additional procedure with associated complications (infection, pneumothorax, bleeding) in placement of the CIED, but are also at increased risk of long-term complications related to right-ventricular pacing, including left ventricle remodeling leading to HF, hospitalizations, and higher 1-year overall mortality $(7,16)$. Risk stratification is therefore particularly important in patients with pre-existing AF undergoing TAVR, as risk of all-cause and cardiac mortality, in addition to bleeding risk, are elevated in such patients (17-18). As there is currently no means to predict which patients will require CIED placement prior to undergoing TAVR, development of a risk prediction model that can also be utilized in those with preexisting AF is necessary, and pre-procedural counseling should be undertaken in all patients undergoing TAVR.

Our model accurately predicted the risk of CIED implantation following TAVR in patients with pre-existing $\mathrm{AF}$. Both this retrospective analysis in patients with pre-existing AF and the original validation cohort from 
the parent study demonstrated good fit for the validation model $(0.652(0.558-0.745)$ vs $0.693(0.627-$ $0.759)$, respectively).

Similar to the parent study, baseline RBBB on ECG was found to be a significant predictor of post-TAVR CIED placement. This study also identified that new-onset LBBB on post-procedural ECG was also associated with a higher likelihood of requiring post-TAVR CIED placement in patients with AF. Placement of a CIED following new-onset LBBB remains controversial, as many patients will have resolution of the LBBB (19). However, new-onset LBBB, especially in patients with post-TAVR ECG QRS duration of > 160 milliseconds, has been associated with higher 1-year mortality. This may be due to a higher risk of sudden cardiac death due to progression of LBBB to complete heart block (CHB) as the TAVR valve compresses the conduction system, or increased risk of cardiac arrhythmias secondary to progression of heart failure and left ventricular remodeling $(7,20-21)$.

Patients with chronic kidney disease (CKD) were also more likely to require CIED placement. Although this was not observed in our original study, in one recent study by Al-Ogaili et al. that looked at a national registry of patients undergoing TAVR, history of CKD was associated with not only a higher likelihood of developing CHB and mortality, but was also an independent predictor for pacemaker implantation following TAVR (adjusted OR $1.32(1.1-1.58)$; p-value $=0.003)(22)$. This may be exacerbated in patients with AF who already have an underlying cardiac arrhythmia. Similarly, those with CKD are more likely to have wide-spread atherosclerosis and calcification, which may involve the conduction system and aortic valve, increasing the risk of conduction disturbances following TAVR (23).

Other risk prediction models have been developed to identify patients at risk for CIED implantation following TAVR (11-12). However, these models require either data from imaging studies or intraprocedural data, while our risk prediction model relies on clinical data that is easily obtained from most medical records pre-procedurally. Our prediction model will allow the TAVR heart team and electrophysiologist to estimate the risk of CIED requirement beforehand. The Emory model, like our model, contains baseline RBBB as a predictive factor, but also includes a history of syncope, baseline QRS durations $\geq 140 \mathrm{~ms}$, and valve oversizing $>16 \%$ identified on pre-procedural multidetector computed tomographic (CT) imaging (11). While the Emory model notably has a stronger AUC in both the derivation and validation cohorts (0.729 and 0.778, respectively), it relies on pre-procedural CT scanning in order to calculate overall PPM implantation risk. Most importantly, the Emory model was only validated in the patient's undergoing TAVR with the Edwards SAPIEN 3 valve, and therefore may not be applicable to patients undergoing TAVR placement with self-expanding valves.

Shivamurthy et al. recently published a study of external validation of their risk prediction model for postTAVR CIED placement originally developed from the National Inpatient Sample (NIS) database (12, 24). This model, primarily composed of baseline ECG features, includes both sinus bradycardia with or without LBBB. Therefore, this model cannot be used in patients with pre-existing AF to determine their overall risk of post-TAVR CIED. While their model demonstrated significantly higher all-cause mortality, any additional risk in patients with pre-existing $\mathrm{AF}$ cannot be determined.

Retrospective validation of our model in patients with pre-existing AF has several implications. As highlighted above, utilization of baseline characteristics and surgical planning enables pre-procedural risk stratification for the possible need for CIED placement. This allows physicians and associated health care providers to have a more informed discussion of risks and benefits with patients prior to undergoing AVR. Similarly, patient's with high risk for CIED placement based on this prediction model who either have transient or lack of post-TAVR conduction disturbances may warrant prolonged outpatient monitoring with a loop recorder or ambulatory ECG monitoring upon discharge.

Notably, in our previously published cohort, pre-existing AF was associated with a decreased risk of postprocedural CIED placement. In a sub-study of the multicenter, randomized control PARTNER trial, patients with pre-existing AF had similar rates of post-TAVR CIED placement to those with pre-existing sinus rhythm (25). While development of AF following TAVR is associated with significantly higher rates of all-cause and 
cardiac death, pre-existing AF may be protective against post-op CIED (17, 18, 26), although this may be subject to sampling bias given the relatively small cohort of patients with AF in each study. Conversely, in the Siontis et al. meta-analysis, requirement for post-procedural PPM was notably higher in individuals with AF in those who received the Medtronic Core Valve (MCRV) versus the Edwards SAPIEN valve (27). This however likely represents the overall higher incidence of PPM implantation associated with the MCRV in general, than a direct correlation with AF.

\section{Study limitation}

There are several limitations to this study. First, this is a single center, retrospective cohort study. Second, while the risk prediction model was originally developed utilizing data from both older and newer generation prostheses, only newer generation valves were included in this sample. However, this also demonstrates that the model is a good fit for the newer generation of valves, and can continue to be used reliably as these are more commonly implanted today. In addition, intraprocedural variability such as valve oversizing and/or deep implantation could have affected our risk prediction. The lack of external validation is recognized too.

\section{Conclusion}

Our risk prediction model accurately predicts the risk of CIED implantation after TAVR in patients with pre-existing AF. To our knowledge, this is the first retrospective validation to demonstrate adequate fitting in patients with pre-existing AF. The model utilizes easily accessible baseline characteristics that does not require intraprocedural or imaging data. Multi-center prospective validation should be undertaken.

\section{TAVR Consortium Members}

Judith A. Mackall, $\mathrm{MD}^{2}$, and Sergio G. Thal, $\mathrm{MD}^{2}$

\section{References}

1. Tsushima T, Nadeem F, Al-Kindi S, et al. Risk Prediction Model for Cardiac Implantable Electronic Device Implantation After Transcatheter Aortic Valve Replacement. JACC: Clinical Electrophysiology . 2020. doi:10.1016/j.jacep.2019.10.020.

2. Leon MB, Smith CR, Mack MJ, et al. Transcatheter or Surgical Aortic-Valve Replacement in Intermediate-Risk Patients. New England Journal of Medicine . 2016;374(17):1609-1620. doi:10.1056/nejmoa1514616.

3. Mack MJ, Leon MB, Thourani VH, et al. Transcatheter Aortic-Valve Replacement with a BalloonExpandable Valve in Low-Risk Patients. New England Journal of Medicine . 2019;380(18):1695-1705. doi:10.1056/nejmoa1814052.

4. Popma JJ, Deeb GM, Yakubov SJ, et al. Transcatheter Aortic-Valve Replacement with a SelfExpanding Valve in Low-Risk Patients. New England Journal of Medicine . 2019;380(18):1706-1715. doi:10.1056/nejmoa1816885.

5. Reardon MJ, Mieghem NMV, Popma JJ, et al. Surgical or Transcatheter Aortic-Valve Replacement in Intermediate-Risk Patients. New England Journal of Medicine . 2017;376(14):1321-1331. doi:10.1056/nejmoa1700456.

6. Agha AM, Burt JR, Beetler D, et al. The Association between Transcatheter Aortic Valve Replacement (TAVR) Approach and New-Onset Bundle Branch Blocks. Cardiology and Therapy . 2019;8(2):357-364. doi:10.1007/s40119-019-0137-2.

7. Auffret V, Puri R, Urena M, et al. Conduction Disturbances After Transcatheter Aortic Valve Replacement. Circulation . 2017;136(11):1049-1069. doi:10.1161/circulationaha.117.028352.

8. Nomura T, Maeno Y, Yoon S-H, et al. Early Clinical Outcomes of Transcatheter Aortic Valve Replacement in Left Ventricular Outflow Tract Calcification: New-Generation Device vs Early-Generation Device. Journal of Invasive Cardiology . 2018;30(11):421-427.

9. Rampat R, Khawaja MZ, Hilling-Smith R, et al. Conduction Abnormalities and Permanent Pacemaker Implantation After Transcatheter Aortic Valve Replacement Using the Repositionable LOTUS Device. JACC: Cardiovascular Interventions . 2017;10(12):1247-1253. doi:10.1016/j.jcin.2017.03.044. 
10. Sager SJ, Damluji AA, Cohen JA, et al. Transient and persistent conduction abnormalities following transcatheter aortic valve replacement with the Edwards-Sapien prosthesis: a comparison between antegrade vs. retrograde approaches. Journal of Interventional Cardiac Electrophysiology . 2016;47(2):143151. doi:10.1007/s10840-016-0145-2.

11. Kiani S, Kamioka N, Black GB, et al. Development of a Risk Score to Predict New Pacemaker Implantation After Transcatheter Aortic Valve Replacement. JACC: Cardiovascular Interventions . 2019;12(21):2133-2142. doi:10.1016/j.jcin.2019.07.015.

12. Vejpongsa $\mathrm{P}$, Zhang $\mathrm{X}$, Bhise $\mathrm{V}$, et al. Risk Prediction Model for Permanent Pacemaker Implantation after Transcatheter Aortic Valve Replacement. Structural Heart . 2018;2(4):328-335. doi:10.1080/24748706.2018.1467067.

13. Arora S, Misenheimer JA, Ramaraj R. Transcatheter Aortic Valve Replacement: Comprehensive Review and Present Status. Texas Heart Institute Journal . 2017;44(1):29-38. doi:10.14503/thij-16-5852.

14. Ruparelia N, Latib A, Kawamoto H, et al. A Comparison Between First-Generation and SecondGeneration Transcatheter Aortic Valve Implantation (TAVI) Devices: A Propensity-Matched SingleCenter Experience. Journal of Invasive Cardiology . 2016;28(5):210-216.

15. Centers for Medicare and Medicaid Services. Decision Memo for Transcatheter Aortic Valve Replacement (TAVR) (CAG-00430N). Available at: https://www.cms.gov/medicare-coveragedatabase/details/nca-decision-memo.aspx?NCAId1/4257. Accessed December 5, 2019.

16. Fadahunsi OO, Olowoyeye A, Ukaigwe A, et al. Incidence, Predictors, and Outcomes of Permanent Pacemaker Implantation Following Transcatheter Aortic Valve Replacement. JACC: Cardiovascular Interventions . 2016;9(21):2189-2199. doi:10.1016/j.jcin.2016.07.026.

17. Sannino A, Gargiulo G, Schiattarella GG, et al. A meta-analysis of the impact of pre-existing and new-onset atrial fibrillation on clinical outcomes in patients undergoing transcatheter aortic valve implantation. EuroIntervention . 2016;12(8). doi:10.4244/eijy15m11_12.

18. Tarantini G, Mojoli M, Windecker S, et al. Prevalence and Impact of Atrial Fibrillation in Patients With Severe Aortic Stenosis Undergoing Transcatheter Aortic Valve Replacement. JACC: Cardiovascular Interventions . 2016;9(9):937-946. doi:10.1016/j.jcin.2016.01.037.

19. Megaly M, Gössl M, Sorajja P, et al. Outcomes after pacemaker implantation in patients with newonset left bundle-branch block after transcatheter aortic valve replacement. American Heart Journal . 2019;218:128-132. doi:10.1016/j.ahj.2019.07.006.

20. Muntané-Carol G, Guimaraes L, Ferreira-Neto AN, et al. How does new-onset left bundle branch block affect the outcomes of transcatheter aortic valve repair? Expert Review of Medical Devices . 2019;16(7):589-602. doi:10.1080/17434440.2019.1624161.

21. Nazif T, George I, Dizon J, et al. Clinical Impact Of New Left Bundle Branch Block In Intermediate Risk Patients Undergoing Tavr: An Analysis Of The Partner Ii Trial And Registries. Journal of the American College of Cardiology . 2018;71(11). doi:10.1016/s0735-1097(18)31536-5.

22. Al-Ogaili A, Fugar S, Okoh A, et al. Trends in complete heart block after transcatheter aortic valve replacement: A population based analysis. Catheterization and Cardiovascular Interventions . 2019;94(6):773-780. doi:10.1002/ccd.28156.

23. Malik J. Heart disease in chronic kidney disease - review of the mechanisms and the role of dialysis access. The Journal of Vascular Access . 2018;19(1):3-11. doi:10.5301/jva.5000815.

24. Shivamurthy P, Vejpongsa P, Gurung S, et al. Validation of scoring system predicting permanent pacemaker implantation after transcatheter aortic valve replacement. Pacing and Clinical Electrophysiology . September 2020. doi:10.1111/pace.13910.

25. Biviano AB, Nazif T, Dizon J, et al. Atrial Fibrillation is Associated with Increased Pacemaker Implantation Rates in the Placement of AoRTic Transcatheter Valve (PARTNER) Trial. Journal of Atrial Fibrillation . 2017;10(1). doi:10.4022/jafib.1494.

26. Chopard R, Teiger E, Meneveau N, et al. Baseline Characteristics and Prognostic Implications of PreExisting and New-Onset Atrial Fibrillation After Transcatheter Aortic Valve Implantation. JACC: Cardiovascular Interventions . 2015;8(10):1346-1355. doi:10.1016/j.jcin.2015.06.010.

27. Siontis GC, Juni P, Pilgrim T, et al. Predictors of Permanent Pacemaker Implantation in Patients 
With Severe Aortic Stenosis Undergoing TAVR. Journal of the American College of Cardiology . 2014;64(2):129-140. doi:10.1016/j.jacc.2014.04.033.

\section{Figure Legends}

Figure 1: Distribution of patients requiring a CIED following TAVR based on the sum of the risk factors using the risk prediction model.

Figure 2: Linear regression model in calibration plot with an $\mathrm{R}^{2}$ of 0.778 and area under the curve of the receiving operating curve of 0.652 .

Table 1: Baseline characteristics of the patients who did or did not require post-procedural CIED. CIED $=$ cardiac implantable electronic device; $\mathrm{CAD}=$ coronary artery disease $\mathrm{PCI}=$ percutaneous coronary intervention; $\mathrm{CABG}=$ coronary artery bypass graft; $\mathrm{SAVR}=$ surgery aortic valve replacement; $\mathrm{ECG}=$ electrocardiogram; LBBB = left bundle branch block; LAFB = left anterior fascicular block; RBBB = right bundle branch block; $\mathrm{LVEF}=$ left ventricular ejection fraction; AVPG = aortic valve pressure gradient; AVA $=$ aortic valve area.

Table 2: Incidence of CIED placement based on the total sum of the risk prediction score.

\section{Figures}

Figure 1

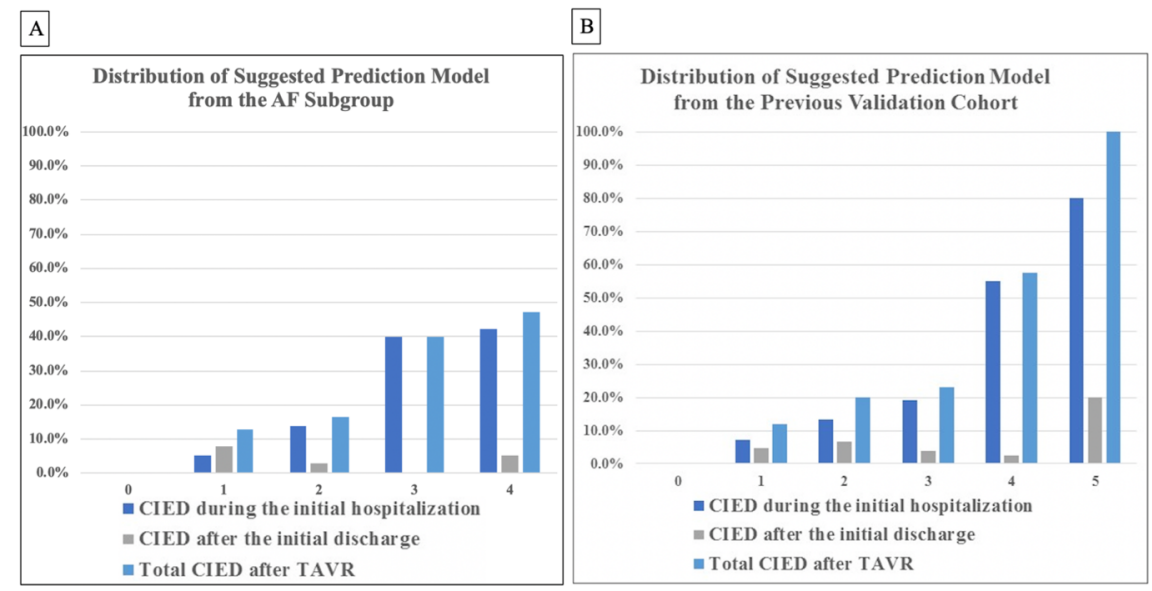

Figure 2 


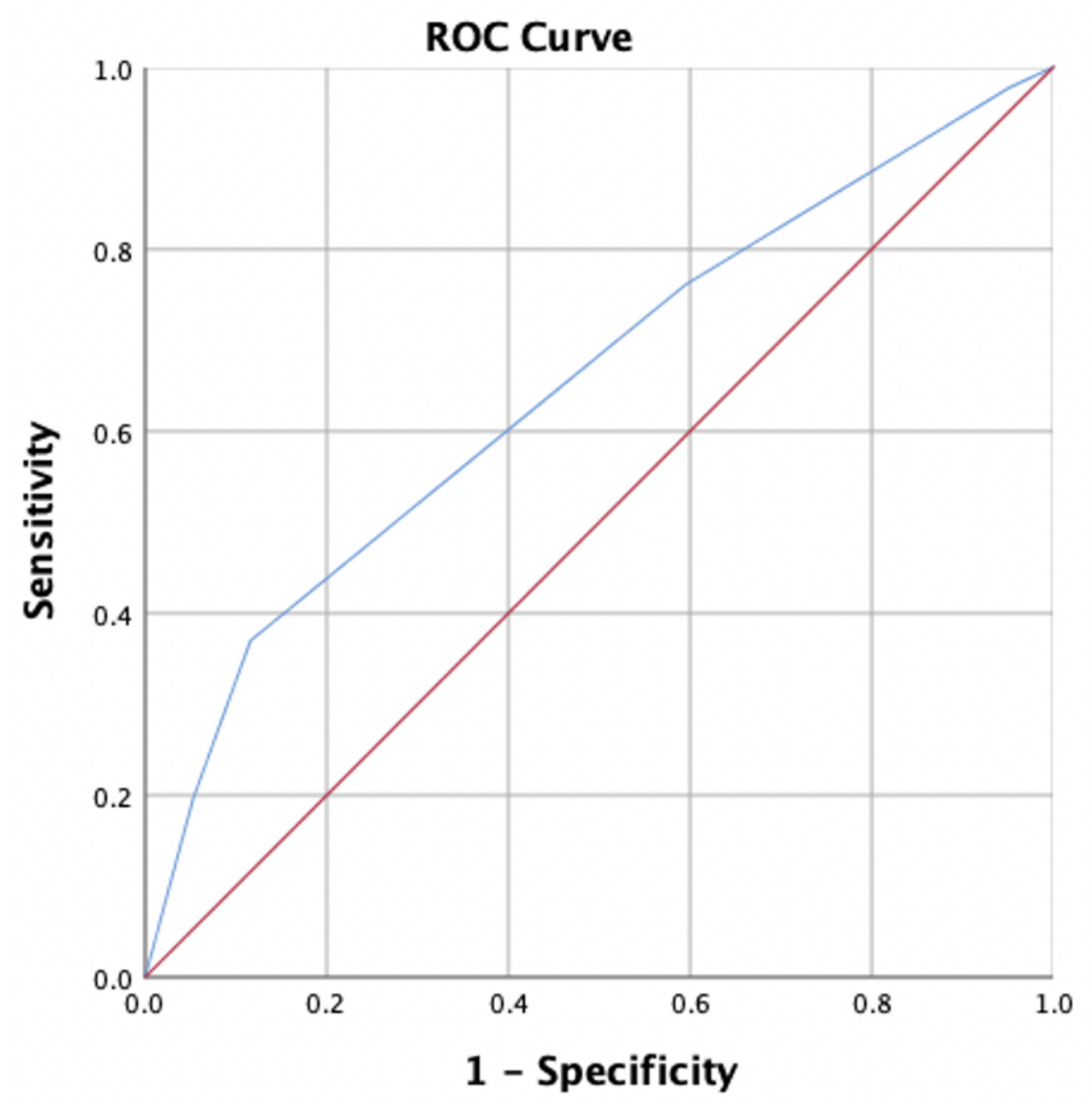

Tables

Table 1

No CIEDs

$(\mathrm{n}=193)$

\section{Baseline}

Characteris-

tics

Male

Age

Hypertension

Diabetes

mellitus

Chronic kidney

disease

Hyperlipidemia 32

Prior stroke 8

Pre-existing 23

CAD

Prior PCI 9

Prior CABG 14
28

$81.38 \pm 9.35$

42

14

27

$68.1 \%$

$17.0 \%$

$48.9 \%$

$19.1 \%$

$29.8 \%$

$59.6 \%$

$89.4 \%$

$29.8 \%$

$57.4 \%$
No CIEDs

$(\mathrm{n}=193)$ p-value
116

26

87

32

28

100

$81.42 \pm 8.11$

173

66

69

6

32

28

$51.8 \%$

0.283

0.690

$89.6 \%$

$34.2 \%$

1.000

0.596

$35.8 \%$

0.003

$60.1 \%$

$13.5 \%$

$45.1 \%$

$16.6 \%$

$14.5 \%$
0.267

0.512

0.452

0.617

0.010 


\begin{tabular}{|c|c|c|c|c|c|}
\hline & CIEDs $(\mathrm{n}=47)$ & CIEDs $(\mathrm{n}=47)$ & $\begin{array}{l}\text { No CIEDs } \\
(\mathrm{n}=193)\end{array}$ & $\begin{array}{l}\text { No CIEDs } \\
(\mathrm{n}=193)\end{array}$ & $\mathrm{p}$-value \\
\hline$\overline{\text { Prior SAVR }}$ & 0 & & 7 & $3.6 \%$ & 0.351 \\
\hline \multicolumn{6}{|l|}{ Baseline } \\
\hline \multicolumn{6}{|l|}{ ECG } \\
\hline LBBB & 2 & $4.3 \%$ & 20 & $10.4 \%$ & 0.264 \\
\hline LAFB & 3 & $6.4 \%$ & 12 & $6.2 \%$ & 1.000 \\
\hline RBBB & 20 & $42.6 \%$ & 23 & $11.9 \%$ & $<0.001$ \\
\hline QRS duration & $116.61 \pm 25.75$ & & $104.84 \pm 26.13$ & & 0.003 \\
\hline \multicolumn{6}{|l|}{ Post TAVR } \\
\hline \multicolumn{6}{|l|}{ ECG } \\
\hline New LBBB & 14 & $29.8 \%$ & 30 & $15.5 \%$ & $<0.001$ \\
\hline QRS duration & $135.93 \pm 31.07$ & & $118.75 \pm 27.01$ & & 0.004 \\
\hline \multicolumn{6}{|l|}{ Baseline } \\
\hline \multicolumn{6}{|l|}{ TTE } \\
\hline LVEF (\%) & $53.59 \pm 12.74$ & & $53.43 \pm 13.54$ & & 0.983 \\
\hline$V \max$ & $3.61 \pm 0.74$ & & $3.92 \pm 0.81$ & & 0.016 \\
\hline Mean AVPG & $32.27 \pm 13.37$ & & $38.42 \pm 15.01$ & & 0.005 \\
\hline $\operatorname{AVA}\left(\mathrm{cm}^{2}\right)$ & $0.77 \pm 0.21$ & & $0.76 \pm 0.27$ & & 0.392 \\
\hline \multicolumn{6}{|l|}{ TAVR } \\
\hline \multicolumn{6}{|l|}{ Procedure } \\
\hline Self-expandable & 24 & $51.1 \%$ & 111 & $57.5 \%$ & 0.424 \\
\hline Valve & & & & & \\
\hline
\end{tabular}

Table 2

\begin{tabular}{llll}
\hline Risk Prediction Score Sum & CIED during initial admission & CIED after initial admission & Total CIED after TAVR \\
\hline 0 & $0.0 \%$ & $0.0 \%$ & $0.0 \%$ \\
1 & $5.1 \%$ & $7.7 \%$ & $12.8 \%$ \\
2 & $13.8 \%$ & $2.8 \%$ & $16.5 \%$ \\
3 & $40.0 \%$ & $0.0 \%$ & $40.0 \%$ \\
4 & $42.1 \%$ & $5.3 \%$ & $47.4 \%$ \\
\hline
\end{tabular}

